\title{
Covid-19: reflexiones filosóficas y gerontológicas desde la adaptabilidad y calidad de vida
}

\author{
Covid-19: philosophical and \\ gerontological reflections from \\ adaptability and quality of life
}

\author{
José Enrique Gómez Álvarez*
}

https://doi.org/10.36105/mye.2021v32n1.04

\section{Resumen}

El objetivo del artículo es mostrar cómo una versión modificada del método de Sgreccia, con las categorías de adaptabilidad y calidad de vida, resulta de utilidad para formular y pensar los problemas de las personas ancianas y adultos mayores en la emergencia del Covid-19. Para lograr lo anterior se retoman los pasos del método bioético: hecho biomédico, valores antropológicos involucrados y respuesta al problema con las categorías de calidad de vida y adaptabilidad. Se analizan los datos empíricos relacionados con la vejez y el Covid-19, mostrando la vulnerabilidad de este grupo frente a la pandemia. Posteriormente, se derivan los deberes éticos que se generan en torno a las personas mayores y ancianas, establecidas por la reflexión y por medio de las categorías. En conclusión, se busca así que las categorías señaladas nos llevan a reconocer la solidaridad y sociabilidad como el eje del equilibrio entre responsabilidad y deber ante los ancianos.

\footnotetext{
* Doctor en Filosofía por la Universidad de Navarra, España. Maestro en Gerontología Social. Profesor investigador de la División de Bioética del Centro de Investigación Social Avanzada. Querétaro, México. Correo electrónico: jegomezalvarez@ yahoo.com https://orcid.org/0000-0002-8964-2207

Recepción: 20 de septiembre de 2020. Aceptación: 15 de octubre de 2020.
} 
Palabras clave: sociabilidad, personas ancianas, prudencia.

\section{Introducción}

La bioética y la gerontología son interdisciplinas y comparten una pregunta común que guía sus reflexiones. La bioética pretende responder a la pregunta de qué es lícito hacer (éticamente) ante determinados estados de la vida humana, entre ellos, los relacionados con la salud y la enfermedad. ${ }^{1}$ Pero la respuesta implica preguntarse eso mismo desde otras disciplinas, como el derecho, las ciencias médicas, el trabajo social y otras.

En el caso de la gerontología sucede algo semejante. La pregunta es: ¿cómo y en qué sentido incrementar la calidad de vida de las personas ancianas? Ese cuestionamiento también es propio de la medicina, la sociología, la ingeniería, el trabajo social, el derecho; de la administración pública y de un largo etcétera de disciplinas que pueden participar en responder esa pregunta.

Ambas, la bioética y la gerontología, tienen varios elementos comunes que las unen. Entre esos conceptos se encuentran el de «calidad de vida» y el «cuidado». La gerontología busca, así, incrementar la calidad de vida en la vejez mediante los cuidados apropiados. Ese aumento de la calidad de vida es visto como un deber individual y social. La bioética también ha utilizado ese término para referirse a las condiciones de vida con pacientes cuya salud está comprometida por distintas dolencias, casos en los que se debe intervenir para incrementar o evitar la disminución de la calidad de vida. Tales intervenciones implican la idea de cuidado.

Este artículo reflexiona heurísticamente sobre los conceptos de adaptabilidad y calidad de vida en relación con la situación vulnerable de los ancianos en el contexto actual de la pandemia de Covid-19 para, así, pensar directrices éticas del deber respecto de ellos y de los deberes de ellos hacia los demás. 
Covid-19: reflexiones filosóficas y gerontológicas desde la adaptabilidad y...

Se trata de una reflexión filosófica, aunque tiene el propósito de convertirse en criterios de operación concreta, y poder generar intervenciones más completas desde la perspectiva de la bioética/gerontología, siguiendo la metodología propuesta por Elio Sgreccia.

\section{Calidad de vida, adaptabilidad y método bioético}

La noción de «calidad de vida» [CV] (1) puede referirse a componentes objetivos y subjetivos, como pueden ser el ingreso económico, el acceso a unidades de salud específicas, la presencia de enfermedades crónicas o cómo se siente una persona ante el balance de los logros en la vida y su percepción del futuro.

...la CV es definida como un estado de bienestar general que comprende descriptores objetivos y evaluaciones subjetivas de bienestar físico, material, social y emocional, junto con el desarrollo personal y de actividades; todas éstas mediadas por los valores personales (1, p. 64).

¿Qué elementos diferenciales se pueden aportar respecto de la situación de la pandemia actual por Covid? Pues un elemento crucial es la adaptabilidad al entorno. La pandemia ha roto ese ambiente más o menos estable entre quienes están libres de peligro de ser afectados en su salud y ocupaciones, y pueden disponerse al cuidado de otras personas. Ha desequilibrado el entorno, al grado de ser todos vulnerables y todos tener un cierto deterioro de la calidad de vida, tal como la hemos definido. Las personas están siendo afectadas en componentes subjetivos de bienestar que, llevados al extremo, significa la pérdida de la salud mental. Esta vulnerabilidad implica la necesidad de adaptación comunitaria e individual. Por las limitaciones funcionales de la vejez, la adaptabilidad se ve más limitada que en el resto de los grupos sociales. Podría argüirse, no obstante, que la mengua de la adaptabilidad es una ventaja, ya que las medidas aplicadas de disminución de movilidad o de confinamiento pueden ser 
más fáciles para las personas que están acostumbradas a «estar en casa» por su relativa menor movilidad.

Por otro lado, puede insistirse en que esto resulta ilusorio. Lamentablemente, aunque el anciano tenga, por ejemplo, una pensión, quizá pueda no salir tanto. No obstante, si presenta enfermedades crónicas, la necesidad de atención médica implica salidas a lugares de mayor riesgo, como puede ser la clínica. También, aunque los ancianos con movilidad restringida permanezcan en sus casas, requieren apoyos «mínimos» para cocinar y realizar el aseo del hogar, lo que les obliga a recibir en sus casas a personas ajenas, con el riesgo implícito de contagio.

Se sugiere utilizar, como se mencionó arriba, el modelo de Sgreccia. Por supuesto que se encuentran otras taxonomías que hacen énfasis en otros valores fundamentales (2). No obstante, he elegido el personalismo de Sgreccia en cuanto que comparte los presupuestos de que hay posibilidad de alcanzar el bien moral como algo objetivo y universalizable (2). También el modelo de decisión del triángulo puede adaptarse a los propósitos de la exposición que se hace aquí. Sgreccia menciona o esquematiza el método bioético del siguiente modo:

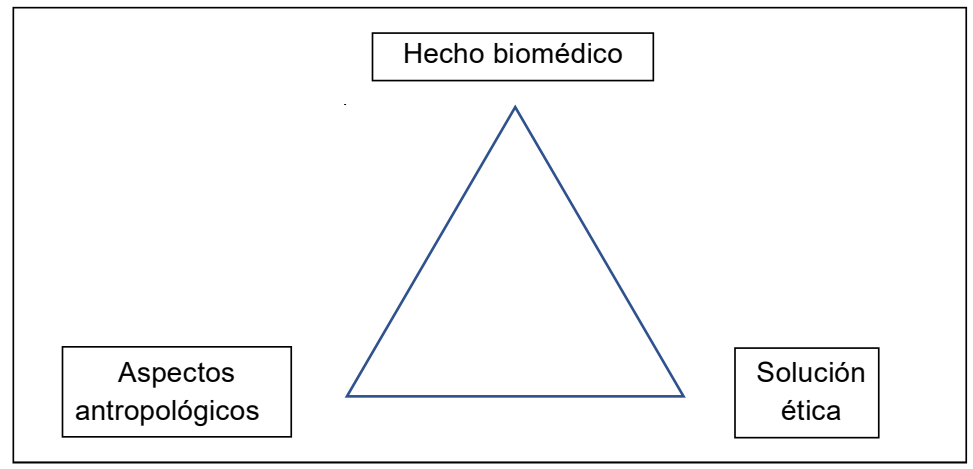

Fuente: Elaboración propia.

El triángulo debe ser entendido en dos dimensiones. Por un lado, una dimensión estática representada por los vértices del mismo, 
Covid-19: reflexiones filosóficas y gerontológicas desde la adaptabilidad y...

que muestran los componentes de cualquier análisis bioético. Estos componentes deben estar presentes de manera simultánea, de modo análogo a como el triángulo sólo cobra forma al estar simultáneamente presentes los tres vértices. La realidad analizada se ve en un prisma interdisciplinario, en donde confluyen o se configura la realidad moral gracias a la perspectiva conjunta del derecho y los datos empíricos, como pueden ser los casos de la medicina (o de la bioética clínica), las realidades sociales e individuales, etcétera. Por otro lado, el triángulo sugiere una visión dinámica del juicio ético, en la que, a partir de una antropología, se obtienen unos principios que sirven para interpretar los hechos que nos dan la pista del correcto actuar ante el caso concreto. Pero a la vez, tenemos que partir del caso concreto, someterlo al tamiz antropológico para descubrir los valores en juego, y de nuevo contrastarlo por la realidad empírica de las ciencias respectivas.

Así, la argumentación ética no es puramente deductiva. La relación interdisciplinaria representa o prepara un juicio práctico para determinar la elección concreta, que es más acorde a los bienes que se tutelan en el caso: «Este juicio de conciencia es... la expresión de la valoración concreta y, en línea de principio, no arbitraria con la que se ordenan jerárquicamente los bienes que se deben tutelar... [para] incrementar o respetar la condición humana» (3, p. 71).

La imagen del triángulo también puede interpretarse como una conexión verdadera en todos los niveles de decisión o reflexión. Dicho de otro modo, como verdades que análogamente se presentan entre todas las disciplinas, lo que permite preguntarse qué cosa sería bueno hacer o no hacer. La verdad se hace presente como una configuración del deber ser: hay verdades en los ámbitos empíricos y normativos que dan pie al juicio práctico de la razón.

Propongo, por tanto, que a la imagen del triángulo se le entrecrucen las dos nociones mencionadas que, en el caso de los ancianos, deben estar presentes en todo el análisis: calidad y adaptabilidad. De este modo, la gráfica quedaría así: 


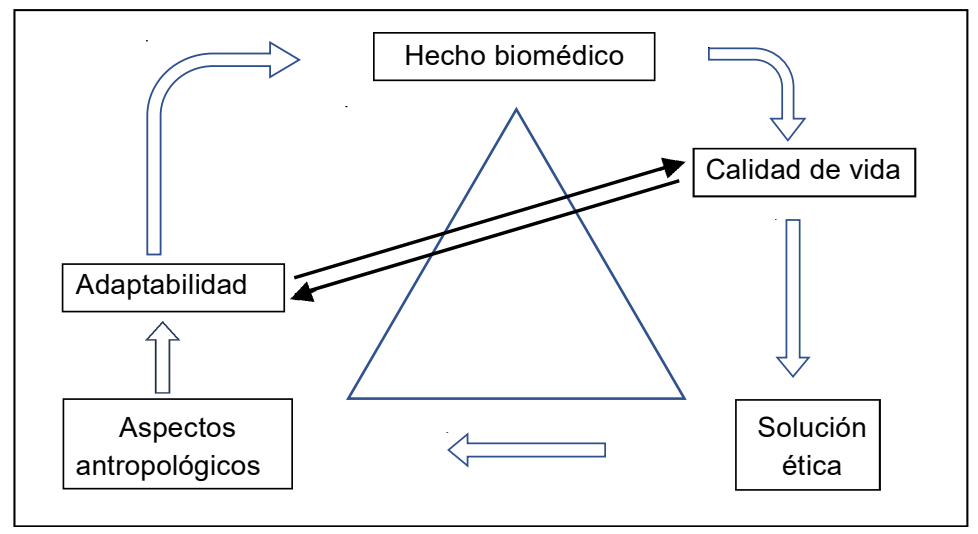

Fuente: Elaboración propia.

A los conceptos antes propuestos podrían añadírseles algunos otros. No obstante, para fines de este análisis sugiero tener a la vista sólo ésos. Las flechas insisten en el aspecto dinámico del triángulo. Así, el hecho (4) biomédico (aunque podría ampliarse a los hechos de intervención límite sobre la vida humana) debe interpretarse en conjunción con la antropología: lo que estudiamos son dos elementos que nos llevan al juicio práctico de la razón, al juicio ético en donde medimos (mediante la prudencia) la adecuación aquí y ahora de esos valores antropológicos y deberes descubiertos. A su vez, los conceptos de adaptabilidad y calidad se entrecruzan. Traducido en términos operativos se podría decir: nos realizamos preguntas acerca de estos conceptos en cada momento del análisis ético. Se produce un círculo virtuoso y no vicioso con el carácter antropológico. Es virtuoso porque es una visión en espiral en donde se regresa al hecho, ya con un juicio realizado para corroborar su correcta pertinencia (6). Sugiero interpretar estos elementos de análisis con las dos categorías que debemos tener siempre presentes. Más adelante se mostrará un cuadro sobre cómo aplicar lo anterior al caso que nos ocupa.

La adaptabilidad no se propone aquí como una especie de concepto evolutivo, como una mera «supervivencia del más fuerte». El 
Covid-19: reflexiones filosóficas y gerontológicas desde la adaptabilidad y...

concepto se ha aplicado en ámbitos como el laboral, en el que se presenta como la capacidad de adaptación a las circunstancias, de modo análogo al modelo sociobiologista, porque la adaptación a las nuevas condiciones del trabajo es indispensable para sobrevivir. Pese a ello, algunas definiciones señalan otros sentidos del término. Por ejemplo: «la adaptabilidad implica una modificación del comportamiento de acuerdo con las circunstancias cambiantes, con el fin de lograr un "ajuste" -la correspondencia entre las circunstancias deseadas y las reales-, así como el ajuste entre los entornos internos y externos de la organización» (7, p. 15).

Entendida así, la adaptabilidad alude a un aspecto del razonamiento práctico de la prudencia: ésta debe ver lo universal o la norma del deber ser general, pero al mismo tiempo debe ver el particular. Debe haber un conocimiento de ambas para así poder hacer el ajuste de una cosa a la otra. La adaptabilidad, pues, implica tener al mismo tiempo el ideal que la razón marca y conocer la situación concreta de ajuste. La adaptabilidad, entendida así, tiene un rasgo comunitario: todos hacemos el ajuste solidario para que se nivelen o se tenga acceso a los bienes básicos que todos merecemos por ser personas. En este último sentido, el concepto de adaptabilidad se aleja de la mera supervivencia «del mejor» mencionada antes; deja de ser un ajuste individual para defenderse de las amenazas del exterior, incluyendo a las otras personas. La adaptabilidad solidaria, pues, posee el rasgo del ajuste universal/particular, en donde vemos no sólo los bienes humanos, sino los demás que se involucran en la génesis, distribución y goce de los mismos.

Lo anterior nos lleva a la siguiente pregunta: ¿son lo mismo adaptabilidad y prudencia? Me parece que no. La prudencia abarca más, ya que en las situaciones ordinarias de la vida, en donde no hay una modificación radical del entorno, no podemos más que aplicar la prudencia para ver la razón del bien de la acción en cada caso concreto de la búsqueda del bien. La prudencia habla de ejecución en situación ordinaria. Considero que la adaptabilidad hace alusión a un grado de ruptura mayor entre la expectativa de los 
acontecimientos y lo que sucede, pero la dirige la prudencia: tenemos que ajustarla siempre en razón del bien que se produce en mí y en los demás.

La adaptabilidad como componente prudencial no puede hacer un cálculo infinito de los singulares: sencillamente no acabaría el cálculo de todas las posibilidades si no se realiza, por decirlo así, una red de semejanzas, en las que vemos similitudes que nos dan patrones cercanos a lo individual (6).

La adaptabilidad, en el sentido comunitario ya señalado, se refiere más bien al ajuste solidario y subsidiario en la relación entre las personas, las cuales, todas entre sí, se deben respeto y cierto amor de benevolencia. Buscamos nuestro bien, no sólo el bienestar, y el bien auténtico de los demás. Así, la adaptabilidad es la creación de las condiciones de encuentro, que reafirman que somos seres personales manifestando nuestra humanidad. A su vez, la adaptabilidad puede analizarse separando sus propósitos: adaptabilidad en las relaciones familiares y de amistad; adaptabilidad en los patrones de consumo; adaptabilidad en las redes de apoyo y en toda una serie de situaciones más. Para poder hacer un juicio ético apropiado, una vez hechas estas reflexiones desde la antropología, revisemos ahora algunos datos empíricos en relación con el Covid y los ancianos.

\section{La pandemia y la afectación en los ancianos: los datos empíricos y la legalidad}

Como se dijo en párrafos anteriores, la noción de «calidad de vida» puede dividirse en aspectos objetivos y subjetivos. El número de fallecidos, por supuesto, es el campo de pérdida radical de la calidad de vida. Hay discrepancia respecto de esa afectación. En Europa, como es sabido, muchos países han tenido claramente defunciones de personas mayores, como España e Italia. No obstante, en Alemania ha sido menor la afectación en ese grupo etario (8). 
Covid-19: reflexiones filosóficas y gerontológicas desde la adaptabilidad y...

No sorprende, dado que Europa cuenta con poblaciones envejecidas. Asimismo, si comparamos lo anterior con China:

...del total de casos que murieron en el país hasta el 11 de febrero de 2020 , el $14,8 \%$ de las personas infectadas por Covid-19 tenía 80 años y más. En Italia, en un análisis que comprendió hasta el 4 de marzo de 2020, el Instituto Nacional de Salud detectó que el promedio de edad de las 105 personas fallecidas por el virus fue de 81 años $(9$, p. 8$)$.

Pero, ¿qué sucede en México? Según los datos del reporte diario de afectados, hasta el 2 de septiembre (10), el total de defunciones acumuladas en México era de 65 mil 816 personas. Lo lamentable es que una proporción alta de personas de más de 60 años han fallecido a causa de la enfermedad, pese a que el porcentaje de adultos mayores en México no se compara con el de España, que es de $20 \%$ (8), mientras que en México es de $12.3 \%$ de la población total (10). El propio INEGI apunta unas características que muestran el problema al que se enfrentan los ancianos en México:

...en el país residen 15.4 millones de personas de 60 años o más, de las cuales 1.7 millones viven solas. Cuatro de cada diez personas de 60 años o más que viven solas $(41.4 \%)$ son económicamente activas. Siete de cada diez (69.4\%) personas de edad que viven solas presentan algún tipo de discapacidad o limitación (11, p. 1).

Así, aunque carecemos de datos que podamos cruzar para saber si las personas que fallecieron vivían solas o tenían una discapacidad, los datos del INEGI proporcionan evidencia acerca de los riesgos de fragilidad en las personas mayores, probablemente incrementados por la pandemia. Lo anterior muestra la exposición y daño que reciben las personas mayores. Aun en casos de personas no fallecidas, pero recuperadas en casa, la necesidad de cuidados puede verse comprometida. Asimismo, es preciso recordar que la mayoría de los adultos mayores en México viven en la viudez (11), lo que implica, por un lado, que se requiere una mayor adaptabilidad de los 
ancianos debido a sus circunstancias de aislamiento y, por otro -a causa de que sus márgenes de acción probablemente estén debilitadas-, que requieren de apoyos de redes sociales para poderse mantener sanos y funcionales. Ante el fenómeno del Covid, incluso en México, donde se dio un relativo confinamiento voluntario, fácilmente los ancianos quedaron doblemente desprotegidos. Primero, al no poder ser atendidos por sus familiares, que debían permanecer «lejos» para protegerlos de la propia epidemia; segundo, ante la imposibilidad de los propios ancianos de apoyarse en redes de su propia edad, debido a la misma situación de aislamiento y a las limitaciones funcionales. Ahora bien, otro dato importante es que las personas mayores de 60 años, en su mayoría viven en regiones de alta vulnerabilidad. Como muestra de contraste: «el mínimo registro de porcentaje de población de 60 años y más fue de $2.5 \%$ en el municipio de García, Nuevo León, y el máximo, de 42\% en Cosoltepec, Oaxaca; la media de todos los municipios fue de 13\% (12, p. 9).

Con la información disponible se puede afirmar que los datos empíricos muestran que, no sólo podemos imaginar conceptualmente a un anciano frágil o expuesto, sino que las propias condiciones sociales antes señaladas dificultan su existencia; al menos, algunos de ellos viven con un riesgo mayor que un adulto más joven.

Lo anterior hace referencia a los hechos, pero debemos considerar, además, que aparte de los deberes éticos con las personas más vulnerables, está la consideración legal, que les reconoce derechos fundamentales exigibles a todos. Por ejemplo, la Constitución de la Ciudad de México establece en el artículo 11, inciso F:

Las personas mayores tienen los derechos reconocidos en esta Constitución, que comprenden, entre otros, el derecho a la identidad, a una ciudad accesible y segura, a servicios de salud especializados y a cuidados paliativos, así como a una pensión económica no contributiva a partir de la edad que determine la ley $(13$, p. 43).

Esta Constitución prevé derechos o acciones que deben fomentarse siguiendo el principio de la sindéresis. Pero también señala as- 
Covid-19: reflexiones filosóficas y gerontológicas desde la adaptabilidad y...

pectos de lo que no debe suceder con ellos, previniendo «...el abuso, abandono, aislamiento, negligencia, maltrato, violencia y cualquier situación que implique tratos o penas crueles, inhumanas o degradantes, o atente contra su seguridad e integridad» (13, p. 43).

Lo anterior, llevado al campo de la atención a los pacientes de Covid-19 de la tercera edad, implica que los grupos organizados, públicos y privados, deben garantizar esas condiciones en la situación actual de la pandemia. Lo jurídico empata con lo ético.

La legislación vigente permite tener criterios para medir la vulnerabilidad de los ancianos respecto del Covid. Esta vulnerabilidad se da en todas las circunstancias en donde les sean privados bienes humanos esenciales, o no les sean proporcionados de modo que generen aislamiento, privación o trato inhumano. La vulnerabilidad priva o elimina la capacidad adaptativa de los ancianos y, en el fondo, de toda la sociedad. En suma, la noción de vulnerabilidad respecto al Covid es:

El nivel de vulnerabilidad de los habitantes ante el efecto potencial del Covid-19 deriva de las características de las personas o grupos en términos de morbilidad, así como de las condiciones sociales, culturales y económicas que influyen en su capacidad para anticipar, hacer frente, resistir y recuperarse de los efectos adversos del coronavirus (10, p. 4).

He subrayado las tres características que nos remiten al concepto de interpretación filosófica llamado adaptabilidad, ya esbozada en la sección I. Se pueden tomar esos tres componentes con lo que hemos señalado del círculo hermenéutico del triángulo de Sgreccia y convertirlo en principios operativos.

\section{Adaptabilidad y vulnerabilidad: reflexiones frente a las personas ancianas}

Probablemente los ancianos están expuestos a una menor capacidad de anticipación, por lo que esa variable de la vulnerabilidad 
debe ser cubierta por los «otros». La idea que subyace en la vulnerabilidad es la presencia de los demás para cubrir las necesidades de las personas mayores en sus ambientes económicos y sociales. Las personas no-ancianas deben cubrir, en la proporción adecuada y suficiente, sólo lo necesario para que los ancianos puedan recuperar sus estrategias de decisión frente a la pandemia.

El amor de benevolencia hacia las personas mayores, y a cualquier otra persona, implica reconocer el bien como una correspondencia: se reconoce el bien que se tutela y al cual se responde, al mismo amor, en libertad y responsabilidad. Debido a que los hombres somos capaces de interpretar la vida de los otros como seres que buscan su auténtico bien, y que puedo reconocerlo también como mío - en nuestro ejemplo del Covid-19, la salud- eso es lo que permite la activación de recursos para nosotros y los demás, en verdadera solidaridad.

El amor de benevolencia no es un bien instintivo de tipo animal de rebaño, en el que, por imitación y preservación de la especie, me cuido y cuido a los otros. De hecho, la rebeldía y resistencia de los individuos a utilizar cubrebocas constituye una prueba indirecta de que el cuidarse no es meramente instintivo para sobrevivir, sino que bien puedo rechazar ese bien para mí a costa de algún otro bien, en este caso la libertad, ya que lo considero superior al de la propia salud. Así, «...el ser humano puede conocer y querer el bien en cuanto tal, el bien en sí y, por ende, el bien del otro en cuanto otro. Y no sólo percibirlo y quererlo, sino procurarlo... construirlo, darle vida» $(15$, p. 24$)$. Los animales no son altruistas estrictamente, sino que ven o perciben los demás en cuanto beneficio o perjuicio de su entorno. Los humanos percibimos la atracción del bien a cualquier costo.

«Hacer frente» a la pandemia significa «le hacemos frente» en plural: se unen sinérgicamente esfuerzos para generar las condiciones de disminución del daño. En el caso de los ancianos, debido a ciertas limitaciones que se presentan en su entorno y redes sociales de apoyo, se les obliga a sustituir parte de los medios porque es su bien, pero, al 
Covid-19: reflexiones filosóficas y gerontológicas desde la adaptabilidad y...

mismo tiempo, este bien sólo puede ser consentido y adaptado a cada caso. Esto es auténtica subsidiariedad (16). Hacemos frente cuando igualamos las condiciones de todos para resolver el problema de salud. La subsidiariedad no es asistencialismo en salud. Al repartir un cubrebocas, me debo hacer la pregunta (ética): ¿qué requisitos materiales mentales, sociales y espirituales requiere esta persona concreta para que esté en la misma condición social de no enfermarse y preservar su salud? Esta pregunta es la que debe guiar el criterio de «resistir».

Lo anterior conlleva un esfuerzo extra: no basta que el que tiene medios para mantenerse durante una cuarentena se proteja aisladamente, sino que debe cooperar para generar las condiciones, si no iguales, sí equivalentes para los demás. Esto no sólo es una actividad o deber gubernamental; es un deber o un bien que percibimos como algo debido desde lo social. Así, la ruptura del egoísmo no es un sentimentalismo barato, sino el reconocimiento del ser de los demás y del bien aparejado al mismo. Es nuestro deber proporcionarle a un anciano, que necesite trabajar fuera de su casa para sobrevivir, las condiciones para que no se exponga, como puede ser el recibirlo o darle los insumos para que ejerza su libertad y las actividades en casa. Insisto, no es sólo cuestión de una renta o de un subsidio pecuniario del gobierno, sino de proporcionar todos los medios en todos los canales y niveles sociales. Por ejemplo, en el ámbito espiritual, es un deber de los demás proporcionarle los medios de acceso a los ritos religiosos y apoyarle con la tecnología necesaria que el anciano podría no saber o poder manejar.

No significa imponer a los demás nuestra visión del bien, sino respetar el bien de los otros, no destruyendo o absorbiendo su capacidad de decisión. Podría uno preguntarse: ¿no es esto realmente algo imposible? ¿Acaso caemos en el asistencialismo al intervenir en la vida del anciano?

La respuesta no es un simple sí o no. De nuevo, es una decisión de la prudencia: se requiere un cierto cálculo para ver si la ayuda proporcionada no está guiada por el interés o la sustitución del otro. 
Se debe correr cierto riesgo ante la libertad de la otra persona: el del rechazo de la ayuda. En este caso, el único camino que queda es la persuasión, como medio para disminuir el riesgo de perder todo ante las debilidades de todos a la hora de darnos condiciones de resistencia.

Pero esta medida es delicada: puede convertirse en asistencialismo; es decir, considerar al necesitado como un menor de edad que sin ti (nosotros) no puede. Este asistencialismo choca con una verdadera asistencia, en la que sólo intervengo estrictamente lo necesario para que la persona recupere su capacidad de reacción y de afrontar y recuperarse.

El hacer frente, resistir y recuperase, señalado en la cita previa, es un desglose de la adaptabilidad. Hacer frente no es «yo me pongo en tu lugar», sino un «te acompaño en el proceso». Así, por ejemplo, lo correcto no es: "anciano no salgas a atender tus necesidades», y yo lo hago en tu lugar por tu debilidad, sino «salgo contigo», sin sustituir la voluntad y autonomía del anciano. Por supuesto, lo anterior no significa abdicar de tomar medidas sanitarias, sino que el anciano asuma los riesgos que considere pertinentes, de modo análogo al de cualquier adulto mayor, evitando el infantilismo y el ageísmo de «someterlo» para «su propio bien».

Por ejemplo, en Colombia se restringió la salida del domicilio a las personas mayores de 70 años, pero el Tribunal Administrativo de Cundinamarca, con una decisión en respuesta a un grupo de quejosos, adultos mayores, «...tumbó las restricciones que les impuso el Gobierno Nacional en razón de la pandemia del Covid-19. Esto quiere decir que, en adelante, esta población podrá salir al aire libre tanto como las personas de 18 a 69 años» (17, s. p.). ¿Qué razones aportó el Tribunal?

Como criterios para suprimir las restricciones, invocó la independencia y autodeterminación, y para evitar el acto discriminatorio con respecto al resto de grupos de adultos. Cabe insistir en que no sólo se trata de autonomía, sino de reconocimiento de la libertad e igualdad de los derechos de los ancianos. El criterio del Tri- 
Covid-19: reflexiones filosóficas y gerontológicas desde la adaptabilidad y...

bunal fue, pues, oponerse a que los ancianos fuesen tratados como menores de edad. Para remediar lo anterior, la subsidariedad y solidaridad sirven para discernir nuestros deberes y derechos con los ancianos. Es un juicio proporcionado por la prudencia, que da su peso a cada elemento de la vida de todos. Por eso es tan complicado, mejor dicho, tan complejo, atender las situaciones de la pandemia, debido a la imposibilidad de la solución única. Por ejemplo, desde el gobierno, la pregunta de «qué hacer con los ancianos» -expresión desafortunada, por cierto-, implica la instrumentalización de sus personas.

Pero no sólo las condiciones de salud y el envejecimiento ponen en peligro a las personas mayores. Retomando los elementos vistos de la soledad y el aislamiento, que se han comprobado empíricamente $(18,19,20)$, es verdad que el sentimiento de soledad y aislamiento como generador estructural están correlacionados, pero, al menos conceptualmente, uno no es causa del otro. Es perfectamente concebible sentir soledad dentro de un hogar o estando acompañado. No obstante, en el caso de los ancianos suele darse al menos cierta correlación. Es crucial combatir la misma para facilitar la adaptabilidad y recuperar la calidad de vida.

El aislamiento no puede sustituirse sólo por medios electrónicos como las videollamadas, ya que su efectividad parece limitada (19), sino que debe incluir un todo integral, en donde los aspectos generales de tener visitas, recibir medicamentos, salir a áreas públicas, entre otras medidas tomadas por la prudencia, deben ajustarse a la realidad concreta, de modo que se recupere o se igualen las condiciones de acceso a los bienes. Así, por ejemplo, para una persona una salida a la semana será suficiente; otra requerirá salir a diario; otros no requieren asistencia en medicamentos, pero otros la necesitan continuamente (como el oxígeno), y un largo etcétera.

Otro modo de encontrar respuestas a las necesidades de las personas ancianas mayores es realizarse las preguntas adecuadas. A continuación, se describe un ejemplo de cómo pueden convertirse en 
preguntas éticas los distintos factores vistos a lo largo de la reflexión:

\begin{tabular}{|c|c|c|}
\hline $\begin{array}{l}\text { Problemas o } \\
\text { hechos } \\
\text { biomédicos }\end{array}$ & Adaptabilidad & Calidad de vida \\
\hline $\begin{array}{l}\text { Aislamiento y } \\
\text { depresión (21). }\end{array}$ & $\begin{array}{l}\text { Hacer frente, resistir, recuperarse. } \\
\text { ¿Qué recursos se disponen para poderle } \\
\text { aislar y por cuánto tiempo? ¿Qué } \\
\text { elementos de las redes sociales pueden } \\
\text { mantenerse como medios de contacto, } \\
\text { como el teléfono u otro medio con una } \\
\text { accesibilidad de ser posible } \\
\text { continua? ¿Es factible tener alguna visita } \\
\text { con todas las medidas de } \\
\text { precaución? ¿Qué recursos se deben } \\
\text { poner a disposición de otros, sin } \\
\text { ponerlos en riesgo de contagio pero } \\
\text { disminuyendo el aislamiento? Si hay } \\
\text { riesgo o presencia de depresión, ¿se } \\
\text { disponen de personas responsables y de } \\
\text { acceso a servicios de emergencia y/o a } \\
\text { personas que puedan auxiliar en una } \\
\text { crisis? ¿Se disponen de protocolos? }\end{array}$ & $\begin{array}{l}\text { ¿Qué criterios utilizar } \\
\text { para determinar que las } \\
\text { interacciones resulten en } \\
\text { verdaderos contactos } \\
\text { personales, y no sólo } \\
\text { informativos del estado } \\
\text { de las personas mayores } \\
\text { recluidas? Si se utilizan } \\
\text { medios convencionales } \\
\text { como el teléfono, ¿qué } \\
\text { puede hacerse para } \\
\text { incrementar la cercanía? }\end{array}$ \\
\hline $\begin{array}{l}\text { Pérdida de } \\
\text { apoyos en redes } \\
\text { sociales para } \\
\text { alimentación y } \\
\text { medicamentos, } \\
\text { entre otros. }\end{array}$ & $\begin{array}{l}\text { ¿Qué medidas se han tomado para } \\
\text { acercar los medicamentos a las } \\
\text { personas? ¿Qué proporción le corres- } \\
\text { ponde al gobierno y qué parte debe } \\
\text { dejarse en manos de las acciones de los } \\
\text { ciudadanos? }\end{array}$ & $\begin{array}{l}\text { ¿Cómo garantizar que no } \\
\text { sólo el acceso, sino los } \\
\text { pacientes que requieren } \\
\text { de alimentación especial, } \\
\text { debido a enfermedades } \\
\text { crónicas, se cubran sin } \\
\text { menoscabo de las } \\
\text { decisiones del anciano y } \\
\text { su especificidad? }\end{array}$ \\
\hline $\begin{array}{l}\text { Consecuencias } \\
\text { de la enferme- } \\
\text { dad: arritmias e } \\
\text { infartos (22). }\end{array}$ & $\begin{array}{l}\text { Asegurar la disponibilidad de equipos } \\
\text { médicos para atender urgencias. No } \\
\text { basta la mera alta de Covid, sino el } \\
\text { seguimiento para evitar el riesgo de } \\
\text { muerte. Por privilegiar el Covid, ¿cómo } \\
\text { suplir, y quién debe hacerlo, el acceso a } \\
\text { recursos de uso continuo, como el } \\
\text { llenado de tanques de oxígeno a } \\
\text { domicilio o el recibir fisioterapia? }\end{array}$ & $\begin{array}{l}\text { ¿Cómo y quiénes deben } \\
\text { intervenir para disminuir } \\
\text { el riesgo de muerte por } \\
\text { falta de atención? }\end{array}$ \\
\hline
\end{tabular}


Covid-19: reflexiones filosóficas y gerontológicas desde la adaptabilidad y...

\begin{tabular}{|c|c|c|}
\hline $\begin{array}{l}\text { Inflamación y } \\
\text { síndrome de } \\
\text { insuficiencia } \\
\text { respiratoria } \\
\text { aguda (22). }\end{array}$ & $\begin{array}{l}\text { ¿Se ha considerado la probabilidad de } \\
\text { requerir esteroides, oxígeno complemen- } \\
\text { tario y broncodilatadores, permitiendo } \\
\text { cierta recuperación de las funciones del } \\
\text { anciano? ¿Quién y cómo se puede } \\
\text { atender la probable pérdida de las } \\
\text { actividades de la vida diaria (AVI), sin } \\
\text { atentar contra la decisión de la persona } \\
\text { mayor? }\end{array}$ & $\begin{array}{l}\text { Se pueden recuperar } \\
\text { funciones de autocuida- } \\
\text { do. ¿Quiénes deben } \\
\text { garantizar el acceso a los } \\
\text { medicamentos? ¿Cómo } \\
\text { distribuirlos sin poner en } \\
\text { riesgo a los involucrados? } \\
\text { En caso de escasez, } \\
\text { ¿quien debe responder } \\
\text { por ellos? }\end{array}$ \\
\hline $\begin{array}{l}\text { Inaccesibilidad } \\
\text { de servicios } \\
\text { públicos } \\
\text { gubernamen- } \\
\text { tales (23). }\end{array}$ & $\begin{array}{l}\text { ¿Es posible, y cómo, implementar } \\
\text { mecanismos de acceso a distancia, } \\
\text { acordes con las habilidades de las } \\
\text { personas ancianas mayores para } \\
\text { trámites indispensables? }\end{array}$ & $\begin{array}{l}\text { Con la persona anciana } \\
\text { mayor, ¿quién debe } \\
\text { sustituirla y hasta cuándo, } \\
\text { por ejemplo, con poderes } \\
\text { para poder resolver sus } \\
\text { asuntos legales? }\end{array}$ \\
\hline $\begin{array}{l}\text { Inaccesibilidad } \\
\text { de servicios de } \\
\text { salud para } \\
\text { enfermedades } \\
\text { no relacionadas } \\
\text { con el Covid. }\end{array}$ & $\begin{array}{l}\text { ¿Las líneas de emergencia han conside- } \\
\text { rado que el aislamiento impide solicitar } \\
\text { un servicio de emergencia? ¿Qué rutinas } \\
\text { deben hacer las personas no ancianas } \\
\text { para cerciorarse diariamente del estado } \\
\text { de las personas mayores ancianas? } \\
\text { Desde la perspectiva del Gobierno, ¿se } \\
\text { ha reservado y es posible parte de los } \\
\text { servicios de urgencia que garantice } \\
\text { acceso eficaz y eficiente a las emergen- } \\
\text { cias no derivadas del Covid? ¿Qué } \\
\text { cuidados pueden y deben transferirse a } \\
\text { asociaciones de ciudadanos y cuáles } \\
\text { deben permanecer exclusivos del } \\
\text { Estado, como, por ejemplo, la ejecución } \\
\text { de pruebas de Covid en personas } \\
\text { ancianas? }\end{array}$ & $\begin{array}{l}\text { ¿Cómo mantener la } \\
\text { calidad de atención, al } \\
\text { tener personal de salud } \\
\text { asignado a la propia } \\
\text { pandemia? }\end{array}$ \\
\hline
\end{tabular}

Fuente: Elaboración propia.

\section{Conclusiones}

El método de Sgreccia permite en sí mismo una visión integral y simultánea de los elementos de decisión ética. No obstante, puede complementarse con otras categorías que enriquecen o permiten 
focalizar mejor las decisiones éticas. Para el caso particular de las personas ancianas, el concepto de calidad de vida y adaptabilidad, muy usados en la gerontología, enriquecen esa metodología y nos ayudan a la reflexión de la pregunta central ética: ¿qué debemos hacer ante las necesidades de las personas ancianas mayores que respete su libertad, pero, a su vez, no las deje en desventaja respecto a personas no ancianas y manteniendo su calidad de vida? La tabla de preguntas éticas trata de dar elementos para mejorar las soluciones respecto de las personas ancianas en esta crisis de la pandemia.

La discusión anterior puede traducirse en la subsidariedad (16). Se deben proporcionar a las personas mayores elementos que no los arriesguen, o los arriesguen sólo en proporción al respeto a sus decisiones autónomas y a los bienes que se tutelan; o sea, siempre con respeto a su libertad y en correspondencia con sus responsabilidades personales y sociales.

La reflexión bioética y gerontológica respecto de la pandemia implica considerar una sinergia en principios éticos, como el de la solidaridad y sociabilidad humanas propias del personalismo, junto con la evidencia empírica que apoye verdaderas intervenciones respetuosas de la dignidad, pero, a su vez, vislumbrando su viabilidad de implementación.

Las preguntas sugeridas sirven para recordarnos lo dicho por Tomás de Aquino sobre el carácter de la prudencia: tenemos que medir, en situaciones como la pandemia, cómo seguimos respetando la dignidad humana, la cual se manifiesta en la capacidad de decidir y ejecutar, dentro de lo posible, las propias decisiones de vida. Asimismo, debemos hacer un cálculo en el que se maximice la calidad de vida, sin atentar contra el principio de proporcionalidad.

El Covid-19 nos recuerda que la reflexión ética ordinaria no sólo no se suple, sino que se amplifica y exige de todos, por el principio de sociabilidad y solidaridad, asumir responsabilidades y derechos de todos los involucrados. En el caso de las personas ancianas ma- 
Covid-19: reflexiones filosóficas y gerontológicas desde la adaptabilidad y...

yores, genera una exigencia de solidaridad que no es optativa, sino el único modo de responder con plenitud humana.

\section{Notas bibliográficas}

${ }^{1}$ La bioética no se reduce a la ética médica, por lo que aborda temas como la ética ambiental, la ingeniería genética y otros.

\section{Referencias bibliográficas}

1. Urzúa, A. et al. Calidad de vida: una revisión teórica del concepto. Terapia psicológica. 2012; (30): 1, 61-71. https://doi.org/10.4067/s0718-48082012000100006 2. Amo U. Bioethical models. Acta bioeth. 2019; 25(1). [Consultado 11 de agosto de 2020]. Recuperado en: https://scielo.conicyt.cl/scielo.php?script=sci_arttext\&pid= S1726-569X2019000100103\&Ing=en. http://dx.doi.org/10.4067/S1726-569X2019 000100103

3. Pessina A. La cuestión del método desde la perspectiva de la bioética personalista. En: Tarasco, M, editora. Comités hospitalarios de bioética. México: Manual Moderno; 2007; 67-74.

4. Aquí hemos de entender «hecho» como «una posibilidad objetiva de verificación, de comprobación o de control y, por lo tanto, también de descripción o de previsión objetiva, en el sentido de que cada uno puede hacerla propia en las condiciones adecuadas» (Abbagnano, N. Diccionario de Filosofía. $1^{\text {a }}$ ed. México: Fondo de Cultura Económica, 1974. El énfasis está dado en lo que podemos denominar realidad accesible a los demás y sujeto a escrutinio.

5. Así que no se entra en discusión con la posición de Hume de la pura factividad. Consideramos que lo normativo está presente en la realidad. Pueden verse, en particular, las páginas 146-153: Beuchot, M. Filosofía y Derechos Humanos. $1^{\text {a }}$ ed. México: Siglo Veintiuno editores; 1993.

6. Aquino T. Suma de Teología. 2012. [Consultado el 14 de agosto de 2020]. Recuperado en: https://hjg.com.ar/sumat/c/c47.html\#a3

7. Foronda F. La adaptabilidad en las organizaciones. Una revisión teórica del concepto y de los elementos que la configuran como una capacidad organizacional [Tesis]. Medellín: Universidad de Antioquía; 2018. Recuperado en: http://biblio tecadigital.udea.edu.co/dspace/bitstream/10495/9952/1/ForondaFrank_2018_ AdaptabilidadOrganizacionesRevision.pdf

8. Jaramillo F, Minué S, Oyarzo L. Covid-19, una lupa sobre la vejez. Cuad Méd Soc. 2020; 60 (1). [Consultado el 21 de agosto de 2020]. Recuperado en: http:// 
cms.colegiomedico.cl/wp-content/uploads/2020/05/CMS12020EspecialPandemia.pdf\#page $=45$

9. Huenchuan S. Covid-19: Recomendaciones generales para la atención a personas mayores desde una perspectiva de derechos humanos (LC/MEX/TS.2020/6/ Rev.1). Ciudad de México, Comisión Económica para América Latina y el Caribe (CEPAL). 2020.

10. Gobierno de México. Covid-19 México. [Consultado el 3 de septiembre de 2020]. Recuperado en: https://coronavirus.gob.mx/datos/\#DOView

11. INEGI. Estadísticas a propósito del día internacional de las personas de edad ( $1^{\circ}$ de octubre). [Consultado 21 de agosto de 2020]. Recuperado en: https://www. inegi.org.mx/contenidos/saladeprensa/aproposito/2019/edad2019_Nal.pdf

12. Suárez L, et al. Índice de vulnerabilidad ante el Covid-19 en México. Investigaciones geográficas. Instituto de Geografía. 2020. Recuperado en: https://www. researchgate.net/profile/Irasema_Alcantara-Ayala/publication/341327204_In dice_de_vulnerabilidad_ante_COVID-19_en_Mexico_Version_completa_en_es panol_e_-p_r_i_n_t_Investigaciones_Geograficas_Instituto_de_Geografia_UN̄AM /links/5ebb2ae8299bf1c09ab92541/Indice-de-vulnerabilidad-ante-COVID-19-enMexico-Version-completa-en-espanol-e-p-r-i-n-t-Investigaciones-Geograficas-Instituto-de-Geografia-UNAM.pdf

13. Instituto de Transparencia, Acceso a la Información Pública, Protección de Datos Personales y Rendición de Cuentas de la Ciudad de México. Constitución de la Ciudad de México. [Consultado el 14 de agosto de 2020]. Recuperado en: http:/ /www.infodf.org.mx/documentospdf/constitucion_cdmx/Constitucion_\%20 Politica_CDMX.pdf

14. Chávez JH, Colín Luna JI, Mendoza Martínez P, et al. Recommendations for the integral treatment of older adults with Covid-19. Med Int Mex. 2020; 36(3). [Consultado el 24 de agosto de 2020]. Recuperado en: https://www.medi graphic. com/cgi-bin/new/resumenl.cgi?IDARTICULO=93812

15. Melendo T. Ámbito natural de la educación: la familia. $1^{\text {a }}$ ed. México: Ed. Trillas; 2008.

16. González E. Resumen de principios fundamentales. En: AAVV. Manual de Doctrina Social Cristiana. México: IMDOSoc; 1989; 213-219.

17. Pessina A. La cuestión del método desde la perspectiva de la bioética personalista. En: Tarasco M, editora. Comités hospitalarios de bioética. México: Manual Moderno. 2007; 67-74.

18. El Tiempo. Definitivo: se cayó la cuarentena forzada para los mayores de 70. [Consultado el 12 de agosto de 2020]. Recuperado en: https://www.eltiempo.com/ justicia/servicios/coronavirus-hoy-adultos-mayores-de-70-anos-podran-salir-a-lacalle-tras-tumbar-restricciones-528328

19. Rodríguez M. La soledad en el anciano. Gerokomos. 2009; 20 (4). [Consultado el 27 de agosto de 2020]. Recuperado en: http://scielo.isciii.es/scielo.php? script=sci_arttext\&pid=S1134-928X2009000400003\&Ing=es 
Covid-19: reflexiones filosóficas y gerontológicas desde la adaptabilidad y...

20. Morel M, Pantoja T, Ortiz Muñoz, LE, Resumen SUPPORT de una revisión sistemática: Videollamadas para reducir el aislamiento social y la soledad en las personas mayores: una revisión rápida. OSF Preprints. 2020. [Consultado el 21 de agosto de 2020]. Recuperado en: https://www.researchgate.net/publication/ 342691390_Resumen_SUPPORT_de_una_revision_sistematica_Video_llamadas _para_reducir_el_aislamientosocial_y_la_soledad_en_las_personas_mayores _una_revision_rapida_-_Resumen_support_EPOC_Chile https://doi.org/10.17605/ OSF.IO/A2VUH

21. Hernández J. La soledad de los ancianos. Temperamentum. 2020; v16. [Consultado 20 de agosto de 2020]. Recuperado en: http://ciberindex.com/index.php/t/ article/view/e12891

22. Ramírez Ortiz J, Castro Quintero D, Lerma Córdoba C, Yela Ceballos F, Escobar Córdoba F. Consequences of the covid-19 pandemic in mental health associated with social isolation. Preprint. 2020. [Consultado el 28 de agosto de 2020]. Recuperado en: https://preprints.scielo.org/index.php/scielo/preprint/view/303/ver sion/310

23. Ponce $L$, Muñiz $T$, Mastarreno $C$, Villacreses $H$. Secuelas que enfrentan los pacientes que superan el Covid-19. RECIMUNDO. 2020; 4(3). [Consultado 28 de agosto de 2020]. Recuperado en: http://www.recimundo.com/index.php/es/article/ view/858 
